\title{
Establishing the eradication unit of Molara Island: a case of study from Sardinia, Italy
}

\author{
Lapo Ragionieri • Giulia Cutuli • Paolo Sposimo • \\ Giovanna Spano • Augusto Navone - Dario Capizzi • \\ Nicola Baccetti · Marco Vannini $\cdot$ Sara Fratini
}

Received: 8 August 2012/Accepted: 4 May 2013/Published online: 17 May 2013

(C) Springer Science+Business Media Dordrecht 2013

\begin{abstract}
Molara is a small island belonging to the Marine protected Area Tavolara-Punta Coda Cavallo, in Sardinia. During 2006-2007, a bio-monitoring program reported a strong presence of the black rat, Rattus rattus, on Molara island. Rat predation has detrimentally affected the unique biodiversity of this island, thus, in 2008 an eradication campaign was conducted. Our eradication protocol included a preeradication genetic investigation, using 8 microsatellite loci, on a rat population of Molara as well as on neighbour islands within the Marine Protected Area (MPA). The main goal of this genetic investigation was to establish the correct borders of the eradication unit of Molara island. As several recent eradication campaigns have been unsuccessful, due to incomplete
\end{abstract}

L. Ragionieri $(\bowtie) \cdot$ G. Cutuli · M. Vannini .

S. Fratini $(\varangle)$

Department of Biology, University of Florence, via

Madonna del Piano 6, 50019 Sesto Fiorentino, Italy

e-mail: lapo.ragionieri@ua.pt

S. Fratini

e-mail: sarafratini@unifi.it

L. Ragionieri

RNA Biology Laboratory, Department of Biology and CESAM, University of Aveiro, 3810-193 Aveiro,

Portugal

P. Sposimo

Nature and Environment Management Operators

srl(NEMO), Follonica, GR, Italy and unstable eradication, we also aimed to assess possible hidden sources of reinvasion. Specimens were also collected during post- eradication monitoring on Molara for genetic screening to establish their origin, and thus validate the effectiveness of our eradication campaign. According to our genetic analysis, within the MPA there are four different eradication units, corresponding to the islands of Molara, Tavolara, Piana and to the Sardinia mainland. Gene flow among these four units is more or less absent. The assignment and clustering tests performed on pre and post-eradication samples seem to indicate that the population of Sardinia mainland is a possible source of re-invasion for the Piana and Molara populations.

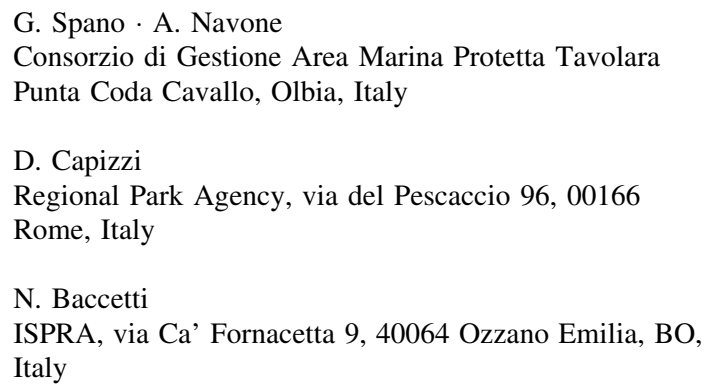


Keywords Population genetics - Eradication campaign - Rattus rattus - Invasive species · Mediterranean Sea

\section{Introduction}

The black rat (Rattus rattus), the Norway rat (Rattus norvegicus) and the Pacific rat (Rattus exulans) are recognised as dangerous worldwide pest (Lowe et al. 2000; King et al. 2011), and during the last decades many eradication campaigns have been conducted, particularly in insular systems and forests, in order to maintain and protect the biodiversity, which the presence of rats may impact. According to these studies, only a few years after these eradications, incredible re-establishment of lost biodiversity has been observed (Towns et al. 2001; Graham and Veitch 2002; Kerbiriou et al. 2004; Pascal et al. 2005; Amaral et al. 2010; Veitch et al. 2011). However, there are also examples of eradication campaigns that were not as successful, and a complete and stable eradication was not achieved (Thorsen et al. 2000; Courchamp et al. 2003; Parkes et al. 2011; Savidge et al. 2012). Various factors may have been responsible for the failure of eradication in these cases, such as the capability of rats to re-invade the same environments, and the presence of some individuals which survived the eradication (Abdelkrim et al. 2007). In fact, the high reproduction rate of rats, coupled with the absence of predators and/ or competitors, can counteract onerous eradication efforts in only a few years (Abdelkrim et al. 2007; Russell et al. 2009a; Russell et al 2009b). For example, within island systems, rats originating from populations located on neighbor islands or mainland sites may re-invade an eradicated island (Russell et al. 2010). For these reasons, in recent years, the "eradication unit" concept (sensu Abdelkrim et al. 2007) has been defined as "the interconnected populations that must be eradicated at the same time to prevent rapid recolonization" (for a more comprehensive view of the notion of eradication unit also see: Robertson and Gemmell 2004; Abdelkrim et al. 2005a, 2007, 2010; Capizzi et al. 2010).

The use of genetic techniques for assessing the geographic boundaries of an eradication unit has risen considerably in recent studies (Abdelkrim et al. 2010; Russell et al. 2010; Savidge et al. 2012). From a genetic perspective, an eradication unit consists of a group of populations among which the gene flow is high enough to genetically homogenize populations. The success of an eradication project thus relies on the removal of all the populations belonging to that eradication unit, to reduce the risk of further reinvasion from interconnected populations (Abdelkrim et al. 2007). Genetic methods are also useful in clarifying the geographic sources of past and future arrivals (Pinceel et al. 2005; Carvalho et al. 2009). Genetic analysis conducted on individuals collected during post-eradication monitoring, for instance, recorded a discrepancy between the low rates of gene flow estimated among rat populations from different islets and the source of reinvasion in eradicated populations (Abdelkrim et al. 2007). The most plausible explanation for this phenomenon arises from a tracking experiment conducted on rats released on islands already populated by conspecifics (Granjon and Ceylan 1989). In this study, all the individuals released on islands, where a well established rat colony was formally present, died of injuries within a few days, suggesting strong role of intra-specific competition. Thus, such behavior of rats suggests caution in the delimitation of the true geographic boundaries of an eradication unit.

The Marine Protected Area Tavolara Punta Coda Cavallo is located in Sardinia, near Olbia, and comprises two main islands (Tavolara and Molara), other islets and 76,09 $\mathrm{km}$ of the Sardinian coastline. In 2006-2007, a bio-monitoring program performed on Molara island reported a strong presence of the black rat $(R$. rattus), which preys on chicks and eggs of nesting seabirds. Consequently, an eradication campaign was conducted on this island in 2008. The eradication protocol included a pre-eradication genetic investigation of rat populations belonging to the islands of Molara, Tavolara, Piana and in the area of Capo Coda Cavallo, on mainland Sardinia. This study, through genotyping 8 microsatellite loci in approximately 30 individuals per population, aimed to investigate the extent of the Molara eradication unit and to establish the level and direction of gene flow among rats populations within the MPA Tavolara Punta Coda Cavallo. Genetic analysis, using the same microsatellite loci, was also performed on two new individuals captured 21 months after the eradication campaign, during the post-eradication monitoring on Molara island. We aimed to assess whether they were 
part of the eradicated population (if the eradication campaign had not been completely successful) or whether they were new arrivals from an unknown source population.

\section{Materials and methods}

Study area and eradication project

Tavolara Punta Coda Cavallo was established as a Marine Protected Area (MPA) in 1997; the MPA comprises 15,000 ha of sea and $40 \mathrm{~km}$ of coastal land, near Olbia, Sardinia (Italy, Fig. 1). The largest islands are Tavolara (600 ha) and Molara (340 ha), with several islets (Piana is the largest, with a surface of 12 ha). The island of Tavolara hosts a NATO military post, and in summer the human presence on this island is quite high, with people travelling daily to the island by a ferry leaving from the village of San Paolo (Sardinia mainland). Conversely, the human presence on Molara island is significantly less, and is mainly tourism based along the coast.

The faunal composition of these islands is typically Mediterranean, with a high species richness of reptiles and invertebrates. Moreover, these islands are a preferred area for sea bird nesting. In 2005 and 2006 a monitoring program was conducted for three species of marine bird: the European shag (Phalacrocorax aristotelis desmarestii), Audouin's gull (Larus

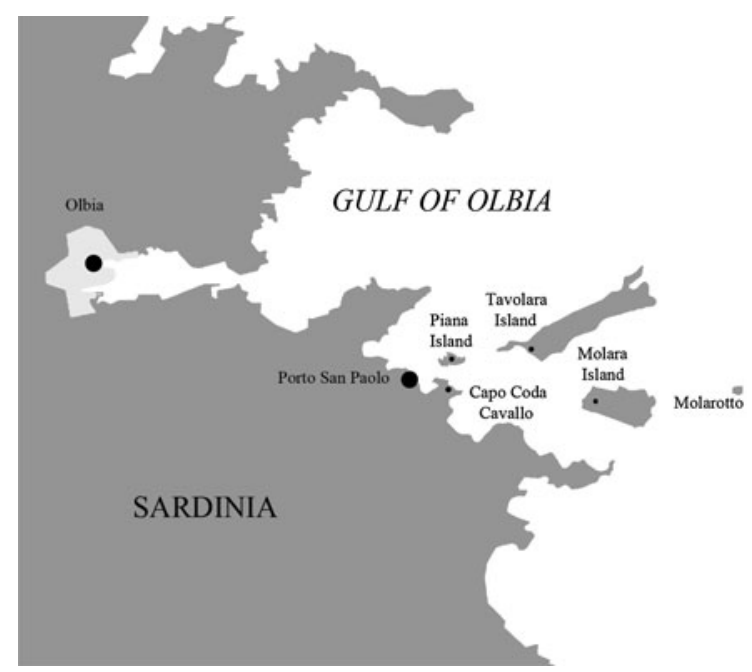

Fig. 1 Map indicating the collection localities of the four population of $R$. rattus audouinii) and the largest global population of Yelkouan shearwater (Puffinus yelkouan) (Zenatello et al. 2011). The island of Molara hosts around 300-600 pairs of Yelkouan shearwaters, that nest among fallen boulders of granite (Baccetti et al. 2009a). Unfortunately, the reproductive success of this species during the monitoring program was estimated to be equal to zero (Sposimo et al. 2012), due to the strong presence of the black rat, which preys heavily on the eggs and chicks of Yelkouan shearwaters. After a preliminary census of rat population density, in 2008, an eradication campaign was initiated on Molara Island. Rodenticide pellets of brodifacoum were spread over the island using a bucket suspended from a helicopter. The effect of the eradication project on Molara was immediately evident as the reproductive success of Yelkouan shearwater in 2009 and 2010 increased (Sposimo et al. 2012). In order to maintain the success of this eradication project, the MPA is planning new eradication projects on neighbor islands.

\section{Sampling collection and DNA extraction}

In order to establish the eradication unit of Molara Island, specimens were collected using bait stations with corns from three islands within the MPA using bait stations with corns along five transects in two different capture session of five consecutive nights: Tavolara Island ( $\mathrm{N}=30)$; Molara $(\mathrm{N}=30)$; Piana $(\mathrm{N}=30)$; and one additional population from Sardinia mainland, Capo Coda Cavallo $(\mathrm{N}=24)$. We also collected two specimens in Molara after eradication. For each captured individual, 10-50 g of tail muscle tissue were preserved in pure ethanol. Then, DNA was isolated using the Puregene Kit (Gentra System), resuspended in TE buffer and then preserved at $-20{ }^{\circ} \mathrm{C}$ for further analysis.

\section{Gene amplification}

Currently, many loci described for Rattus norvegicus and related species (Jacob et al. 1995) are available, and from these we selected eight microsatellite loci used in similar studies (Abdelkrim et al. 2005b, 2009). For detection of polymorphisms, six out of eight primer combinations were divided into two different sets based on similar annealing temperatures and different fragments length (Set R1: D10Rat20, D5Rat83, D7Rat13; Set R2: D9Rat13, D11Mgh5, 
D16Rat8) for multiple PCRs. The two sets of loci were amplified in a Perkin Elmer 9,600 thermal cycler using master mix (Quiagen) by PCR mixture in $15 \mu \mathrm{L}$ of final volume containing: $1 \mu \mathrm{L}$ of DNA, $3.5 \mu \mathrm{L}$ of master mix and $0.3-0.8 \mu \mathrm{L}$ of primers $10 \mu \mathrm{M}$; the PCR cycling conditions were: 35 cycles with $30 \mathrm{~s}$ for denaturation at $95{ }^{\circ} \mathrm{C}, 90 \mathrm{~s}$ for annealing at $57^{\circ} \mathrm{C}$ and $60 \mathrm{~s}$ for extension at $72{ }^{\circ} \mathrm{C}$, preceded by $15 \mathrm{~min}$ of initial denaturation at $95^{\circ} \mathrm{C}$, and followed by $10 \mathrm{~min}$ of final extension at $72{ }^{\circ} \mathrm{C}$. The remaining two loci (D19Mit2 and D10Mit5) were separately amplified by PCR mixture in $20 \mu \mathrm{L}$ of final volume containing: $1 \mu \mathrm{L}$ of DNA, $2 \mu \mathrm{L}$ of buffer 10X (Invitrogen), $2 \mathrm{mM}$ of $\mathrm{MgCl} 2,0.5 \mu \mathrm{L}$ of primers $10 \mu \mathrm{m}, 200 \mu \mathrm{m}$ of each dNTPs and $0.4 \mathrm{U}$ of Taq (Invitrogen); the PCR cycling conditions were: 35 cycles with $30 \mathrm{~s}$ for denaturation at $94{ }^{\circ} \mathrm{C}, 45 \mathrm{~s}$ for annealing at $57{ }^{\circ} \mathrm{C}$ and $60 \mathrm{~s}$ for extension at $72{ }^{\circ} \mathrm{C}$, preceded by $10 \mathrm{~min}$ of initial denaturation at $94{ }^{\circ} \mathrm{C}$ and followed by $30 \mathrm{~min}$ of final extension at $72{ }^{\circ} \mathrm{C}$. For each locus, the forward primer was $5^{\prime}$-labeled with a fluorescent dye of the three different fluorophores (6-Fam, Hex and Ned).

For each set, $3.5 \mu \mathrm{L}$ of each PCR product obtained with master mix was mixed with $1.5 \mu \mathrm{L}$ of PCR product from single locus PCR (Set R1 + D19Mit2; Set R2 + D10Mit5) and combined with water in a final volume of $10 \mu \mathrm{L}$ for successive dimensional analysis. Sizing was performed in an ABI Prism 310 Genetic Analyzer (Applied Biosystems) with reference to an internal size standard (ROX400) using GENOTYPER ver. 3.7 (Applied Biosystems).

Genetic diversity, population genetic structure and bottlenecks

The number of alleles and the allelic richness for each locus and population were calculated using FSTAT ver. 2.9.3 (Goudet 1995). We estimated the Nei's standard genetic distance (Nei 1978) using Microsatellites analyzer 4.05 (Dieringer and Schlötterer 2002). Linkage Equilibrium among loci and Hardy-Weinberg equilibrium (HWE) were assessed for each population using GENEPOP ver. 3.4 (Raymond and Rousset 1995). We used the software MICROCHECKER 2.2.3 (van Oosterhout et al. 2004) to evaluate whether heterozygote deficiencies could be explained by the existence of null alleles.

We estimated the genetic differentiation among populations using the Exact test of population differentiation (Raymond and Rousset 1995), as implemented in GENEPOP. This test verifies the existence of differences in allele frequencies at each locus and for each population. Single locus $p$ values were calculated using a Markov chain with 1,000 batches and 1,000 iterations per batch, combined over loci using the Fisher method.

The existence of population genetic structure was also assessed by one level AMOVA (Excoffier et al. 1992), using ARLEQUIN ver. 3.11 (Excoffier et al. 2005). Significance of the fixation indices, under the null hypothesis of no differentiation among populations, was tested using a non-parametric permutation approach with 10,000 permutations.

In addition, the spatial analysis of molecular variance (SAMOVA) was used to define groups of populations that are geographically homogenous and maximally differentiated from each other as implemented in SAMOVA 1.1 (Dupanloup et al. 2002). The aim of this approach is to define groups of populations $(\mathrm{K})$, which maximize the proportion of total genetic variance due to differences among groups of populations (Fct), by means of an annealing procedure.

We used STRUCTURE version 2.3 (Pritchard et al. 2000) to infer population genetic structure. This Bayesian cluster method takes a sample of genotypes and uses the assumption of HWE and linkage disequilibrium within sub-populations to find the number of populations $(K)$ that fits the data best and the individual assignments which minimize HWE and linkage disequilibrium in those populations. We used the admixture model which is the most appropriate for populations that may have recent ancestors from more than one population (Pritchard et al. 2000). Likelihood of model was assessed by the number of possible clusters $(K)$ ranging between 1 and 4 . A further analysis was performed including the two samples collected on MOL during the post eradication monitoring, with $\mathrm{K}$ ranging between 1 and 5 . We performed five independent runs using an admixture model with allele frequencies correlated. Each run consisted of 1,000,000 iterations (conducted three times for each $\mathrm{K}$ value) with the first 100,000 iteractions discarded as burn-in.

In order to determine the most probable origin of the individuals captured in different locations and during the post-eradication monitoring, we used an assignment method as implemented in GENECLASS 2.0 software (Piry et al. 2004). This method calculates 
the likelihood of the multi-locus genotype of a given individual in a set of pre-determined populations. We chose an assignment threshold of 0.05 and obtained the rejection probability by simulating 10,000 individuals from allelic frequencies. We used the Bayesian method proposed by Rannala and Mountain (1997) and simulation algorithm of Paetkau et al. (2004). The mean probability values were estimated for each individual and population.

We used the software Bottleneck 1.2.02 (Cornuet and Luikart 1997) to determine if the four populations of $R$. rattus underwent a recent bottleneck. This software is based on the principle that populations which have recently experienced a reduction in their effective population size exhibit a corresponding reduction of the allele numbers $(\mathrm{k})$ and gene diversity at polymorphic loci. Usually the number of alleles is reduced faster than the gene diversity. Thus, in a recently bottlenecked population, the observed gene diversity is higher than the gene diversity expected at equilibrium (Heq) which is computed from the observed number of alleles $(\mathrm{k})$, under the assumption of a constant-size (equilibrium) population (Luikart et al. 1998). For detecting if populations underwent genetic bottlenecks we applied the heterozygosity excess method of Luikart et al. (1998) using the Twophased model (TPM), with $70 \%$ of single-step mutations and $30 \%$ of multi-step mutations, and the Stepwise Mutation Model (SMM) as mutation models with 10,000 iterations. We ran Bottleneck using two different statistical tests, the Sign test and the Wilcoxon sign-rank test (Cornuet and Luikart 1996; Luikart and Cornuet 1997; Luikart 1997), both based on 10,000 replications. Bottlenecked populations are also expected to exhibit a characteristic 'mode shift' in the frequency distribution of alleles away from the L-shaped distribution expected under mutation-drift equilibrium (Luikart et al. 1998). Consequently, BOTTLENECK was also used to generate a qualitative descriptor of whether the observed allele frequencies at each locus deviate from such a distribution.

\section{Gene flow among populations}

In order to estimate the gene flow among the four populations, we used MIGRATE 3.2.14 (Beerli and Felsenstein 1999). Input data were converted to Migrate format using Microsatellites analyzer 4.05 (Dieringer and Schlötterer 2002). Migrate estimates the mutation-scaled effective population size Theta $(\theta=\mathrm{xNe} \mu$, where $\mathrm{x}$ is a multiplier depending on the ployd phase $\mathrm{x}=4$ for nuclear data, Ne is the effective population size and mu is the mutation rate per site per generation $\mu$ ) and the mutation-scaled migration rate $\mathrm{M}(\mathrm{m} / \mu$, where $\mathrm{m}$ is the immigration rate and $\mu$ the mutation rate), which is a measure of the importance of immigration in bringing new variants into the population. The effective number of immigrants per generation was estimated by multiplying $\theta \times \mathrm{M}$ (as the equation $\mathrm{N}_{\mathrm{e}} \mathrm{m}_{\mathrm{ji}}=\theta_{\mathrm{i}} \times \mathrm{M}_{\mathrm{ji}}$ ). This analysis produces values of $\theta \times \mathrm{M}$ (4Nem for microsatellites) estimated in each direction among the four populations with their approximate $95 \%$ confidence intervals (Beerli and Felsenstein 2001). We ran MIGRATE three times using a Singlestep Model with mutation rates estimated for each locus, uniform prior distribution $(\theta$ distribution: minimum $=0.0, \quad$ maximum $=20.0$, mean $=10.0 ; \mathrm{M}$ distribution: minimum $=0.0$, maximum $=100.0$, mean $=50.0$ ), starting parameters based on Fst calculations, burn-in equal to 10,000 trees, and 10 replicates. Finally the overall number of migrants per generation (Nem) was estimated by summing $\theta \mathrm{M}$ in each direction and dividing by four for microsatellites (Wright et al. 2005).

\section{Results}

Genetic diversity, population genetic structure and bottlenecks

All loci, except one (D10Rat20), were informative, and presented a relatively high level of polymorphism. The locus D10Rat20 had a high number of null alleles in all populations, and an excess of homozygotes, according to MICROCHECKER; therefore, this locus was removed from subsequent analysis and the overall analyses were conducted using seven out of eight loci. Similar problems for this locus were reported in other studies (Abdelkrim al. 2010; King et al. 2011).

No significant linkage disequilibrium was recorded across all populations. This was expected since the microsatellite loci employed in this study are located on different chromosomes (Jacob et al. 1995).

The mean number of alleles per locus was 10; the populations of MOL and TAV had a similar number of alleles, while the population of PIA had the lowest number of alleles and the CCC population was the 
most polymorphic. In addition, the three populations of MOL, TAV and CCC showed quite a high number of private alleles, those alleles that are present in just one population ( 8,7 and 19 respectively), while the population of PIA was the only population without any private alleles.

The values of expected and observed heterozygosis was higher in the populations of MOL, TAV and CCC, and comparatively lower in the population of PIA (Table 1). The populations of TAV, CCC and PIA deviated from HWE, while the population of MOL was the only one in HWE (Table 1). In order to test if an excess of homozygotes was due to the presence of null alleles at different loci, we employed the software MICROCHECKER. An excess of homozygotes was recorded for the population of PIA due to the locus D19Mit2 and the presence of two loci monomorphic, D7Rat13 and D11Mgh5. The population of TAV presented three loci out of the HWE, D19Mit2 and D11Mgh5 with an excess of homozygotes, and D10Mit5 with an excess of heterozygotes. Similar results were recorded with Fis index for the populations of PIA (in locus D19Mit2) and TAV (for locus D19Mit2 and D11Mgh5), while no significant values were recorded in the populations of MOL and CCC.

The Nei's standard genetic distance, corrected for a small sample size, produced similar values of pairwise genetic divergence for the comparisons involving the three populations of TAV, MOL and CCC (Table 2). The smallest value of genetic divergence was recorded between the populations of PIA and TAV, compared to the other pairwise comparisons.

The AMOVA test recorded a high value of population partitioning ( $F s t=0.328, P<0.001$ ). This was also evident from the pairwise Fst values among the four populations (Table 2); all of the populations were strongly differentiated from each other. Similar results were also recorded with the exact test of population differentiation (data not shown).

The results of the SAMOVA were in agreement with those of the AMOVA; the number of population groups that maximised the distribution of genetic variation was $K=4$ (data not shown). The cluster analysis conducted with the program STRUCTURE recorded the presence of four groups of populations $(\mathrm{K}=4)$, each corresponding to one of the four analysed populations. Furthermore, in this analysis we also included the genotypes of the two samples of $R$. rattus collected on MOL during the post- eradication monitoring. These two samples clustered within the CCC population and not with the MOL samples collected before the eradication campaign (Fig. 2).

The results of the assignment analysis also supported the presence of four independent groups. All the individuals collected from the populations of TAV, MOL and CCC were assigned to their own population (Fig. 3). A small percentage of PIA individuals were assigned to the CCC population, and notably the two rats collected on MOL during the post-eradication monitoring were unambiguously assigned to the population of CCC (Fig. 3).

The analysis conducted with Bottleneck recorded an heterozygosity excess in five out of the seven loci for the population of PIA (Sign test under TPM, $P=0.036$; Wilcoxon test under TPM, $P=0.015$ ), while the remaining two loci were monomorphic. In the populations of TAV and MOL, six loci showed a heterozygosity deficiency, and one locus a heterozygosity excess (TAV: Sign test under SMM, $P=0.02$; Wilcoxon test under the SMM, $P=0.039$. MOL: Sign test under SMM, $P=0.021$; Wilcoxon test under the SMM, $P=0.039$ ). Finally, the population of CCC showed a heterozygosity deficiency (Wilcoxon TEST under the SMM, $P=0.019$ ). These results accord with the allele frequency distribution, which was a normal L-shaped distribution in the three populations of TAV, MOL and CCC, while a shifted distribution of allele frequencies, typical of populations which experienced a bottleneck, was recorded for the population of PIA.

\section{Gene flow}

The four populations of $R$. rattus had low values of effective population size, with the PIA population and CCC population having the smallest and largest values respectively (Table 3 ). The gene flow among the four populations was very weak, without any evidence of asymmetric gene flow. Regarding the overall migration, all the values were weak and without clear evidence of any preferential migration channel for gene flow between pairs of populations (Table 3).

\section{Discussion}

The four populations of $R$. rattus collected in the Marine Protected Area Tavolara Punta Coda Cavallo 
Table 1 Rattus rattus collection localities and summary statistics

\begin{tabular}{|c|c|c|c|c|c|c|c|c|c|c|c|}
\hline \multirow[t]{3}{*}{ Locus } & \multirow{3}{*}{$\begin{array}{l}\text { GPS } \\
\text { Range }\end{array}$} & \multicolumn{5}{|c|}{ Piana (PIA) } & \multicolumn{5}{|c|}{ Tavolara (TAV) } \\
\hline & & \multicolumn{5}{|c|}{$40^{\circ} 53^{\prime} 17.17^{\prime \prime} \mathrm{N} ; 9^{\circ} 39^{\prime} 7.08^{\prime \prime} \mathrm{E}$} & \multicolumn{5}{|c|}{$40^{\circ} 53^{\prime} 36.64^{\prime \prime} \mathrm{N} ; 9^{\circ} 41^{\prime} 2.56^{\prime \prime} \mathrm{E}$} \\
\hline & & $\mathrm{Na}$ & $\mathrm{Ar}$ & Ho & $\mathrm{He}$ & Fis & $\mathrm{Na}$ & $\mathrm{Ar}$ & Ho & $\mathrm{He}$ & Fis \\
\hline D10Rat20 & $114-128$ & - & - & - & - & - & - & - & - & - & - \\
\hline D5Rat83 & $169-195$ & 3 & 3 & 0.6 & 0.559 & -0.08 & 4 & 3.97 & 0.567 & 0.555 & -0.02 \\
\hline D7Rat13 & $153-189$ & 1 & 1 & 0 & 0 & - & 8 & 6.83 & 0.833 & 0.727 & -0.15 \\
\hline D9Rat13 & $112-130$ & 2 & 2 & 0.3333 & 0.2825 & -0.18 & 2 & 1.57 & 0.033 & 0.033 & 0.00 \\
\hline D11Mgh5 & $234-286$ & 1 & 1 & 0 & 0 & - & 4 & 3.58 & 0.172 & 0.533 & 0.68 \\
\hline D16Rat81 & $146-174$ & 3 & 3 & 0.767 & 0.658 & -0.17 & 5 & 4.56 & 0.766 & 0.673 & -0.14 \\
\hline D19Mit2 & $195-235$ & 5 & 4.54 & 0.5 & 0.704 & 0.29 & 7 & 6.05 & 0.466 & 0.74 & 0.37 \\
\hline D10Mit5 & $185-195$ & 2 & 2 & 0.2667 & 0.2825 & 0.06 & 3 & 3 & 0.733 & 0.616 & -0.19 \\
\hline Mean & & 2.4 & 2.4 & 0.352 & 0.355 & 0.01 & 4.7 & 4.22 & 0.51 & 0.554 & 0.08 \\
\hline \multirow[t]{3}{*}{ Locus } & GPS & \multicolumn{5}{|c|}{ Molara (MOL) } & \multicolumn{5}{|c|}{ Capo Coda Cavallo (CCC) } \\
\hline & & \multicolumn{5}{|c|}{$40^{\circ} 52^{\prime} 10.49^{\prime \prime} \mathrm{N} ; 9^{\circ} 42^{\prime} 54.78^{\prime \prime} \mathrm{E}$} & \multicolumn{5}{|c|}{$40^{\circ} 52^{\prime} 45.14^{\prime \prime} \mathrm{N} ; 9^{\circ} 38^{\prime} 59.63^{\prime \prime} \mathrm{E}$} \\
\hline & Range & $\mathrm{Na}$ & $\mathrm{Ar}$ & Ho & $\mathrm{He}$ & Fis & $\mathrm{Na}$ & $\mathrm{Ar}$ & Ho & $\mathrm{He}$ & Fis \\
\hline D10Rat20 & $114-128$ & - & - & - & - & - & - & - & - & - & - \\
\hline D5Rat83 & $169-195$ & 4 & 3.58 & 0.552 & 0.558 & 0.01 & 7 & 6.9 & 0.833 & 0.804 & -0.04 \\
\hline D7Rat13 & $153-189$ & 6 & 5.58 & 0.69 & 0.796 & 0.14 & 8 & 7.38 & 0.75 & 0.702 & -0.07 \\
\hline D9Rat13 & $112-130$ & 4 & 3.25 & 0.172 & 0.165 & -0.05 & 7 & 6.34 & 0.875 & 0.761 & -0.15 \\
\hline D11Mgh5 & $234-286$ & 4 & 3.56 & 0.483 & 0.477 & -0.01 & 10 & 8.94 & 0.667 & 0.824 & 0.19 \\
\hline D16Rat81 & $146-174$ & 4 & 3.97 & 0.552 & 0.554 & 0 & 8 & 7.33 & 0.833 & 0.7332 & -0.14 \\
\hline D19Mit2 & $195-235$ & 8 & 7.43 & 0.62 & 0.783 & 0.21 & 7 & 6.82 & 0.75 & 0.812 & 0.08 \\
\hline D10Mit5 & $185-195$ & 4 & 3.58 & 0.345 & 0.482 & 0.29 & 3 & 3 & 0.294 & 0.4332 & 0.33 \\
\hline Mean & & 4.9 & 4.42 & 0.488 & 0.545 & 0.11 & 7.1 & 6.67 & 0.715 & 0.724 & 0.01 \\
\hline
\end{tabular}

For each population: GPS coordinates, the size range in base pairs of each locus (range), total number of alleles for each locus (Na), allelic richness per locus (Ar), observed heterozygosity (Ho), expected unbiased heterozygosity (He), within population inbreeding coefficient $\left(F_{\text {is }}\right)$. In bold significant of $P$ value of departure from the Hardy-Weinberg equilibrium

Table 2 Pairwise comparisons of genetic differentiation, estimated from the pairwise Fst values (under the diagonals; significant values are in bold; $P$ threshold $<0.05$ ), and Nei's standard genetic distance of the four populations of Rattus rattus (over the diagonal)

\begin{tabular}{lllll}
\hline & PIA & TAV & MOL & CAV \\
\hline PIA & - & 0.398 & 0.572 & 0.495 \\
TAV & $\mathbf{0 . 3 4}$ & - & 0.514 & 0.553 \\
MOL & $\mathbf{0 . 4 4}$ & $\mathbf{0 . 3 2}$ & - & 0.483 \\
CAV & $\mathbf{0 . 3 4}$ & $\mathbf{0 . 2 6}$ & $\mathbf{0 . 2 5}$ & - \\
\hline
\end{tabular}

were genetically differentiated, based on summary statistics (AMOVA and SAMOVA) and clustering and assignment methods. Based on these genetic analyses, we thus recorded four independent eradication units with extremely reduced or absent gene flows.
Otherwise, a small fraction of individuals captured on PIA were assigned to the population of CCC, supporting the theory of a recent invasion of this island by a few individuals from the Sardinian mainland. Although these two populations are separated by a considerable distance $(\sim 1.1 \mathrm{~km})$, there are many small islets between the Sardinian mainland and PIA, such as Isola dei Cavalli, which may have acted as a bridge for sporadic migration events. In Isola dei Cavalli, for instance, rats and mice were present, and they were eradicated at the same time of PIA in a successive eradication campaign conducted between December 2009 and January 2010. Isola dei Cavalli is distant around $200 \mathrm{~m}$ from CCC and around $300 \mathrm{~m}$ from PIA and these distances can be potentially covered by black rats (Abdelkrim et al. 2009; Russell et al. 2009a; 2010; Savidge et al. 2012). Moreover, the 


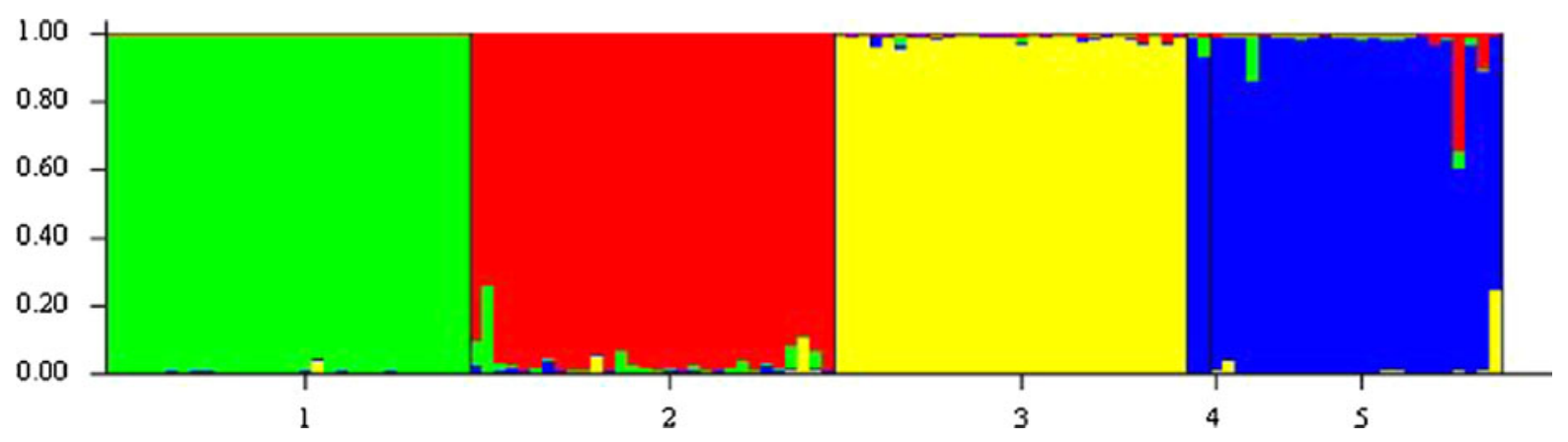

Fig. 2 Cluster analysis: each individual is represented by a vertical bar, with $\mathrm{K}$ colours, where $\mathrm{K}$ is the number of predefined populations and the length of the segments correspond to the individual membership to each population. The run with the highest posterior probability corresponds to $K=4$.

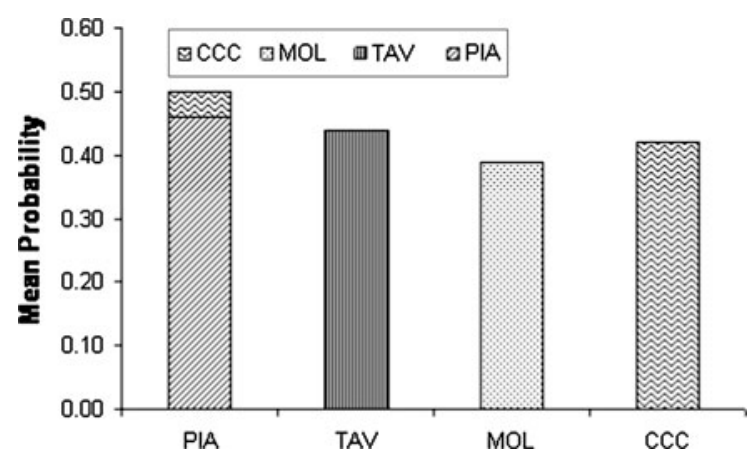

Fig. 3 Assignment test: mean probability values of individuals assigned per population (PIA, Piana; TAV, Tavolara; MOL, Molara; Capo Coda Cavallo, CCC)

fact that PIA and CCC populations were genetically independent could be a consequence of the strong genetic reduction usually associated with recent founding events, which may increase the genetic divergence from the source population (Abdelkrim et al. 2005b). This hypothesis is also supported by the relatively small heterozygosity value, from the reduced number of alleles (two out of seven loci are monomorphic and no private alleles) and the departure from the HWE recorded in PIA population, typical of recent founder events.

The allelic richness, heterozygosity levels and the number of private alleles recorded in the two populations of MOL and TAV were quite similar. The only difference recorded being that the population of MOL was at the HWE, while the population of TAV was out of the equilibrium, due to an excess of homozygotes at two loci. This deviation from HWE could be ascribed to the collection of individuals which were not
Black vertical bars delineate predefined populations (Group 1, PIA in green; Group 2, Tavolara, in red; Group 3 MOL, in yellow; Group 4, two MOL individuals collected after the eradication campaign, in blue; Group 5, CCC, in blue)

representative of the overall population. The population of TAV was, in fact, exclusively collected in an area close to the touristic harbour, because access on the island is restricted to this area due to the presence of a NATO military base.

The population of CCC has comparatively higher levels of genetic variability than the other populations, twice the number of private alleles and a greater population size; however, the CCC population was not in the HWE, with an overall excess of homozygotes. This could be due to the presence in the mainland population of CCC of sub (family) groups (Wahlund effect) or inbreeding effect.

An insular colonization generally involves few individuals and produces effects similar to a genetic bottleneck. After a genetic bottleneck, the observed heterozygosity may exceed the expected heterozygosity as a consequence of a faster reduction of allelic diversity than of heterozygosity (Cornuet and Luikart 1996). At the same time, a modal shift in the distribution of alleles is generally observed together with a relative deficit of rare alleles (Luikart et al. 1998). This is essentially what we observed in the population of PIA. An heterozygosity excess was observed in five out of seven loci, in addition to a shift in the distribution of alleles, typical of populations which have recently undergone a bottleneck or a founding event. On the contrary, in the other three sampled populations, no clear signal of a recent founder or bottleneck event was evident, based on the heterozygosity indexes and on the allele frequency distribution. The three populations of MOL, TAV and CCC appear to have been founded sufficiently long 
Table 3 Effective population size and gene flow among the four populations of $R$ rattus, in the Marine Protected Area of Capo Coda Cavallo, using MIGRATE

\begin{tabular}{lllllll}
\hline & Theta & Pop 1 & Pop 2 & $N_{e} \mathrm{~m}_{12}$ & $N_{e} \mathrm{~m}_{21}$ & $N_{e} \mathrm{~m}$ \\
\hline PIA & $0.14(0.00-0.37)$ & PIA & TAV & $0.25(0.00-1.26)$ & $0.23(0.00-1.39)$ \\
TAV & $0.23(0.00-0.52)$ & PIA & MOL & $0.29(0.00-1.60)$ & $0.30(0.00-1.34)$ & 0.12 \\
MOL & $0.21(0.00-0.49)$ & PIA & CCC & $0.25(0.00-1.81)$ & $0.06(0.00-0.53)$ & 0.15 \\
CCC & $0.30(0.00-0.61)$ & TAV & MOL & $0.33(0.00-1.77)$ & $0.30(0.00-1.60)$ & 0.16 \\
& & TAV & CCC & $0.30(0.00-1.34)$ & $0.33(0.00-1.77)$ & 0.19 \\
& & MOL & CCC & $0.30(0.00-1.45)$ & $0.18(0.00-0.93)$ & 0.12 \\
\hline
\end{tabular}

Theta effective population size (2.5-97.5\% confidence intervals), $\mathrm{N}_{\mathrm{e}} \mathrm{m}_{12}$ mean of migrants from population 1 (Pop 1) to population 2 (Pop 2), and corresponding confidence interval (2.5-97.5\%), $\mathrm{N}_{\mathrm{e}} \mathrm{m}_{21}$ are mean migrants in the opposite direction from Pop 2 to Pop 1 and $N_{e} \mathrm{~m}$ is the effective number of migrants exchanged per generation

ago to become genetically independent, and without any further gene flow connecting these three populations.

In a recent study conducted on a western Mediterranean insular system, Lavezzi island and its surrounding islets, Abdelkrim et al. (2009) reported similar levels of genetic diversity in populations resulting from an ancient colonization event to those recorded in the two rat populations of MOL and TAV, supporting the idea that these two rat populations were well established in the respective islands. Anyway is not possible to evaluate how old were the colonization events on MOL and TAV as far rats seems to be able to established populations with demography and population genetic structure similar to longer established populations, without short-time genetic consequence (Russell et al. 2009b). The population of CCC, indeed, had considerably higher values of genetic diversity, suggesting that this population is considerably larger than any other rat population collected in the Mediterranean Sea, or at least is part of a larger population present on Sardinia mainland. Finally, the genetic diversity recorded in PIA is much less than in all the other populations, with values similar to those recorded from Abdelkrim et al. (2009) on the small islets surrounding Lavezzi Island. In addition, all the alleles present in PIA were also found in the other populations without any private alleles. Even here, all these evidences strongly support a recent origin of PIA probably from the surrounding populations such as $\mathrm{CCC}$ and TAV.

Based on our analysis, it is not possible to assess if the population of CCC is the source population of the MOL and TAV populations, or if the rat populations founded on TAV and MOL islands are the product of a single or multiple invasion events. Rats may have reached the islands swimming or, more likely, through secondary vectors. However, it is also possible that the strong intra-specific behavioral competition, typical of rats, may have limited the survival and establishment of new settlers (Granjon and Ceylan 1989). Moreover, the two rats collected during the post-eradication monitoring on MOL clustered with the Sardinian mainland population (CCC), and not with the preeradication MOL population. Although rats are capable swimmers (e.g. R. norvegicus, Russell et al. 2010), and in some areas are considered one of the most likely invaders of offshore islands (e.g. New Zealand, Russell et al. 2005), the minimum geographic distance between the Sardinia mainland and the island of Molara $(\sim 1.5 \mathrm{~km})$ exceeds the known swimming capability of the black rat (Russell et al. 2010; Calmet et al. 2001). Thus, according to our genetic analyses the eradication campaign on MOL island appears to have been successful, and it is highly probable that the rapid re-invasion of $R$. rattus of MOL was driven by tourist or private boats arriving from Sardinia.

Although the populations of $R$. rattus analyzed in this study appear to be genetically independent, these results must be interpreted with caution for two reasons. Firstly, in the past rats were able to invade and establish permanent populations on all of the major neighbor islands to MOL. Secondly, according to our data, the Sardinia population is a possible source of re-invasion for the islands of MOL (rats were collected during post-eradication monitoring) and PIA (cluster and assignment analysis), and consequently for the entire study area. These evidences suggest that there could be a rat exchange between the Marine Protected Area Tavolara Capo Coda Cavallo and the 
Sardinia mainland, probably driven by humans. The eradication campaign of MOL appears to have been successful thus far; an increase in the reproductive success of Yelkouan shearwater has been observed (Sposimo et al. 2012). Therefore, to maintain the success of this campaign, post eradication monitoring should be coupled with more strict bio-security measures (see Russell et al. 2008), and further eradication campaigns on other islands within the MPA.

\section{Outlook}

In the last decade the use of genetic information acquired from hypervariable autosomal markers, such as microsatellites, became a fundamental prerequisite of many eradication campaigns of pest species. Notwithstanding numerous studies successfully established eradication units, these studies also highlighted some weaknesses of this approach. In first instance, as already reported from Savidge et al. (2012), genetic methods may not always be valid if the populations did not reach the equilibrium conditions for such analysis.

The identification of source population, as in this study, remains a difficult task as well as the ability of discriminating between eradication failure and recolonization events (Russell et al. 2009a; Savidge et al. 2012): this is especially true for mainland populations, with genetically continuous populations, where few local variations may be due to reduced gene flows or to the presence of family groups (Abdelkrim et al. 2010; King et al. 2011). In addition if low local variation is recorded, clustering methods completely fail to identify possible groups of populations (Abdelkrim et al. 2010).

Another critical point is that the true level of gene flow may be underestimated owing to behavioural mechanisms of rats, such as strong intra-specific competition and migrant exclusion (Granjon and Cheylan 1989), in such a situation genetic methods may not be able to record the true gene flow and once the eradicated population is removed, new colonists from interconnected populations may spread rapidly (Abdelkrim et al. 2007).

At the light of the above-mentioned shortcomings, we auspicate that future eradication campaigns will integrate pre-eradication genetic investigations with eco-ethological studies in order to shed new light on the population structure of pests as well as on behavioural mechanisms which can limit the reliability of the genetic tool.

Acknowledgments We are grateful to Massimo Putzu for help with rat sampling. We also thank Jenny Booth for the accurate linguistic revision of the manuscript. We thank two anonymous reviewers for their helpful comments. This research was partially supported by Fondi d'Ateneo to M. Vannini (ex $60 \%$ University of Florence).

\section{References}

Abdelkrim J, Pascal M, Samadi S (2005a) Island colonization and founder effects: the invasion of the Guadeloupe islands by ship rats (Rattus rattus). Mol Ecol 14:2923-2931

Abdelkrim J, Pascal M, Calmet C, Samadi S (2005b) Importance of assessing population genetic structure prior to eradication of invasive species: examples from insular Rattus norvegicus populations. Cons Bio 19:1509-1518

Abdelkrim J, Pascal M, Samadi S (2007) Establishing causes of eradication failure based on genetics: case study of ship rat eradication in ste. Anne Archipel Cons Bio 3:719-730

Abdelkrim J, Pascal M, Samadi S (2009) Genetic structure and functioning of alien ship rat populations from a corsican micro-insular complex. Biol Invasion 11:473-482

Abdelkrim J, Byrom AE, Gemmell NJ (2010) Fine-scale genetic structure of mainland invasive Rattus rattus populations: implications for restoration of forested conservation areas in New Zealand. Conserv Genet 11:1953-1964

Amaral J, Almeida S, Sequeira M, Neves V (2010) Black rat Rattus rattus eradication by trapping allows recovery of breeding roseate tern Sterna dougallii and common tern S.hirundo populations on Feno Islet, the Azores. Portugal. Cons Evidence 7:16-20

Baccetti N, Capizzi D, Corbi F, Massa B, Nissardi S, Spano G, Sposimo P (2009) Breeding shearwater on Italian islands: population size, island selection and co-existence with their main alien predator. Riv Ital Ornitol 78:83-99

Beerli P, Felsenstein J (1999) Maximum-Likelihood estimation of migration rates and effective population numbers in two populations using a coalescent approach. Genetics 152:763-773

Beerli P, Felsenstein J (2001) Maximum likelihood estimation of a migration matrix and effective population sizes in $n$ subpopulations by using a coalescent approach. Proc Natl Acad Sci USA 98:4563-4568

Calmet C, Pascal M, Samadi S (2001) Is it worth eradicating the invasive pest Rattus norvegicus from Molene archipelago? Genetic structure as a decision-making tool. Biodivers Conserv 10:911-928

Capizzi D, Baccetti N, Sposimo P (2010) Prioritizing rat eradication on islands by cost and effectiveness to protect nesting seabirds. Biol Conserv 143:1716-1727

Carvalho DC, Oliveira DAA, Santos JE, Teske P, Beheregaray LB, Schneider H, Sampaio I (2009) Genetic characterization of native and introduced populations of the neotropical cichlid genus Cichla in Brazil. Genet Mol Biol 32:601-607 
Cornuet JM, Luikart G (1996) Description and power analysis of two tests for detecting recent population bottlenecks from allele frequency data. Genetics 144:2001-2014

Cornuet JM, Luikart G (1997) Description and power analysis of two tests for detecting recent population bottlenecks from allele frequency data. Genetics 144:2001-2014

Courchamp F, Chapuis JL, Pascal M (2003) Mammal invaders on islands: impact, control and control impact. Biol Rev 78:347-383

Dieringer D, Schlötterer C (2002) Microsatellite analyser (MSA): a platform independent analysis tool for large data set. Mol Ecol Notes 3:167-169

Dupanloup I, Schneider S, Excoffier L (2002) A simulated annealing approach to define the genetic structure of populations. Mol Ecol 11:2571-2581

Excoffier L, Smouse PE, Quattro JM (1992) Analysis of molecular variance inferred from metric distances among DNA haplotypes: application to human mitochondrial DNA restriction data. Genetics 131:479-491

Excoffier L, Laval LG, Schneider S (2005) Arlequin, version 3: an integrated software package for population genetics data analysis. Evol Bioinform 1:47-50

Goudet J (1995) FSTAT, version 1.2. A computer program to calculate F-statistics. J Hered 86:485-486

Graham MF, Veitch CR (2002) Changes in birds numbers on Tiritiri Matangi Island, New Zealand, over the period of rat eradication. In Veitch CR, Clout MN (eds) Turning the tide: the eradication of invasive species. Occas Pap of the IUCN Species Surviv Comm 27:120-123

Granjon L, Ceylan G(1989) Le sort de rats noirs (Rattus rattus) introduits sur une île, révélé par radio-tracking. C R Acad Sci Paris 571-575

Jacob HJ, Brown DM, Bunker RK et al (1995) A genetic linkage map of the laboratory rat, Rattus norvegicus. Nat Genet 9:63-69

Kerbiriou C, Pascal M, Le Viol I, Garoche J (2004) Consequence sur l'avifaune terrestre de l'Ile de Trielen (reserve naturelle d'Iroise; Bretagne) de l'eradication du surmulot (Rattus norvegicus). Revue d'Ecologie (Terre and Vie) 59:319-329 (in French)

King CM, Innes JG, Gleeson D, Fitzgerald N, Winstanley T, O'Brien B, Bridgman L, Cox N (2011) Reinvasion by ship rats (Rattus rattus) of forest fragments after eradication. Biol Invasions 13:2391-2408

Lowe S, Browne M, Boudjelas S (2000) 100 of the world's worst invasive alien species. A selection from the global invasive species database, Invasive Species Specialist Group

Luikart G (1997) Usefulness of molecular markers for detecting population bottlenecks and monitoring genetic change. University of Montana, Dissertation

Luikart G, Cornuet JM (1997) Empirical evaluation of a test for identifying recently bottlenecked populations from allele frequency data. Conserv Biol 12(1):228-237

Luikart G, Allendorf FW, Cornuet JM, Sherwin WB (1998) Distortion of allele frequency distributions provides a test for recent population bottlenecks. J Hered 89:238-247

Nei M (1978) Estimation of average heterozygosity and genetic distance from a small number of individuals. Genetics 89(3):583-590

Paetkau D, Slade R, Burden M, Estoup A (2004) Genetic assignment methods for the direct, real-time estimation of migration rate: a simulation-based exploration of accuracy and power. Mol Ecol 13(1):55-65

Parkes J, Fisher P, Forrester G (2011) Diagnosing the cause of failure to eradicate introduced rodents on islands: brodifacoum versus diphacinone and method of bait delivery. Cons Evidence 8:100-106

Pascal M, Siorat F, Lorvelec O, Yésou P, Simberloff D (2005) A pleasing consequence of Norway rat eradication: two shrew species recover. Divers Distrib 11:193-198

Pinceel J, Jordaens K, Van Houtte N, Bernon G, Backeljau T (2005) Population genetics and identity of an introduced terrestrial slug: Arion subfuscus s.l. in the North-East USA (Gastropoda, Pulmonata, Arionidae). Genetica 125: 155-171

Piry S, Alapetite A, Cornuet JM, Paetkau D, Baudouin L, Estoup A (2004) GeneClass2: a software for genetic assignment and first generation migrants detection. $J$ Hered 95:536-539

Pritchard JK, Stephens M, Donnelly P (2000) Inference of population structure using multilocus genotype data. Genetics 155:945-959

Rannala B, Mountain JL (1997) Detecting immigration by using multilocus genotypes. Proc Natl Acad Sci USA 94: 9197-9201

Raymond M, Rousset F (1995) GENEPOP ver. 1.2: population genetics software for exact tests and ecumenicism. J Hered 4:248-249

Robertson BC, Gemmell NJ (2004) Defining eradication units in pest control programmes. J Appl Ecol 41:1032-1041

Russell, JC, Towns DR, Clout MN (2008): Review of rat invasion biology: implications for island biosecurity. Science for Conservation 286. Department of Conservation, Wellington $53 \mathrm{p}$

Russell JC, Towns DR, Anderson SH, Clout MN (2005) Intercepting the first rat ashore. Nature 437:1107

Russell JC, Mackay JWB, Abdelkrim J (2009a) Insular pest control within a metapopulation context. Biol Conserv 142:1404-1410

Russell JC, Abdelkrim J, Fewster RM (2009b) Early colonisation population structure of a Norway rat island invasion. Biol Invasions 11:1557-1567

Russell JC, Miller SD, Harper GA, MacInnes HE, Wylie MJ, Fewster RM (2010) Survivors or reinvaders? Using genetic assignment to identify invasive pests following eradication. Biol Invasions 12:1747-1757

Savidge JA, Hopken MW, Witmer GW, Jojola SM, Pierce JJ, Burke PW, Piaggio AJ (2012) Genetic evaluation of an attempted Rattus rattus eradication on Congo Cay, U.S. Virgin Islands, identifies importance of eradication units. Biol Invasions 14:2343-2354

Sposimo P, Spano G, Navone A, Fratini S, Ragionieri L, Putzu M, Capizzi D, Baccetti N (2012) Rodent eradication on Molara Island and surrounding islets (NE Sardinia): from success to the riddle of reinvasion. Aliens 32:33-38

Thorsen M, Shorten R, Lucking P, Lucking V (2000) Norway rats (Rattus norvegicus) on Fregate Island, Seychelles: the invasion; subsequent eradication attempts and implications for the island's fauna. Biol Conserv 96:133-138

Towns DR, Daugherty CH, Cree A (2001) Raising the prospect for a forgotten fauna: a review of 10 years of conservation effort for New Zealand reptiles. Biol Conserv 99:3-16 
Van Oosterhout C, Hutchinson WF, Wills DPM, Shipley P (2004) MICROCHECKER: software for identifying and correcting genotyping errors in microsatellite data. Mol Ecol Notes 4:535-538

Veitch CR, Clout MN, Towns DR (2011) Island Invasives: eradication and management. In Proceedings of the international conference on island invasives. Gland, Switzerland, IUCN and Auckland, New Zealand, CBB. xii $+542 p p$
Wright T, Rodriguez A, Fleischer R (2005) Vocal dialects, sexbiased dispersal, and microsatellite population structure in the parrot Amazona auropalliata. Mol Ecol 14:1197-1205

Zenatello M, Spano G, Baccetti N, Zucca C, Navone A, Putzu M, Azara C, Trainito E, Ugo M, Phillips R (2011) Movements and moving population estimates of Yelkouan shearwaters at Tavolara, Sardinia. In 13th MEDMARAVIS Pan-Mediterranean symposium, Alghero (Sardinia) 14-17 (in press) 